\title{
Valor de la PAAF transrectal en el diagnóstico del cáncer de próstata en pacientes de edad avanzada
}

\author{
Planelles Gómez J, Beltrán Armada JM, Alonso Hernández S, Tarín Planes M, Beamud Cortés M, \\ Gil Salom M.
}

Servicio de Urología. Hospital Universitario Dr. Peset. Valencia.

Actas Urol Esp. 2008; 32(5):485-491

\begin{abstract}
RESUMEN
VALOR DE LA PAAF TRANSRECTAL EN EL DIAGNÓSTICO DEL CÁNCER DE PRÓSTATA EN PACIENTES DE EDAD AVANZADA

Objetivos: Identificar si existe algún grupo de varones ancianos con sospecha clínica de cáncer de próstata, en el cual la confirmación anatomopatológica del diagnóstico puede no ser necesaria, y valorar si la punciónaspiración con aguja fina (PAAF) transrectal de próstata puede ser adecuada para el diagnóstico en la población anciana.

Material y método: Se evaluaron mediante PAAF transrectal a 72 pacientes de edades comprendidas entre 75 y 93 años. No fue necesaria la administración de ningún tipo de profilaxis antibiótica, analgesia, ni el abandono o sustitución de medicación anticoagulante.

Resultados: En 35 de estos pacientes (48,6\%) el resultado de la citología fue positivo para adenocarcinoma de próstata, mientras que en 37 fue negativo para tumor. En el 100\% de los pacientes con PSA > $30 \mathrm{ng} / \mathrm{ml}$ o con PSA $>20 \mathrm{ng} / \mathrm{ml}$ y tacto rectal sospechoso de cáncer, la PAAF fue positiva. Se detectó un 4,1\% de complicaciones menores y un $1,3 \%$ de complicaciones mayores (prostatitis aguda).

Conclusiones: En varones mayores de 75 años con PSA > $30 \mathrm{ng} / \mathrm{ml}$ o con PSA > $20 \mathrm{ng} / \mathrm{ml}$ y tacto rectal sospechoso puede no ser necesaria la confirmación histológica de cáncer de próstata mediante biopsia dada la elevada probabilidad de que ésta sea positiva. Cuando se considere necesaria la confirmación anatomopatológica del diagnóstico de cáncer de próstata en pacientes de edad avanzada, la PAAF constituye una buena alternativa a la biopsia transrectal, dada su excelente tolerancia y baja tasa de complicaciones.
\end{abstract}

Palabras clave: Cáncer de próstata. Ancianos. PAAF. Biopsia. PSA.

\section{ABSTRACT}

VALUE OF TRANSRECTAL FNA IN THE DIAGNOSIS OF PROSTATE CANCER IN ELDERLY PATIENTS

Objectives: To identify if there is a group elderly patients with clinical suspicion of prostate cancer in which pathological confirmation may be unnecessary, and if prostatic transrectal fine needle aspiration (FNA) may be a useful diagnostic tool for old patients

Material and methods: A total of 72 patients aged 75 - 93 years were evaluated by means of prostatic transrectal FNA. Antibiotic prophylaxis, analgesia or cessation of anticoagulant therapy were not necessary.

Results: In 35 patients (48.6\%) cytological diagnosis was positive for prostatic adenocarcinoma, whereas in 37 cytology was negative for cancer. In $100 \%$ of patients with PSA $>30 \mathrm{ng} / \mathrm{ml}$ or with PSA $>20 \mathrm{ng} / \mathrm{ml}$ and suspicious digital rectal examination FNA results were positive for cancer. $4.1 \%$ minor and 1.3\% major (acute prostatitis) complications after FNA were observed

Conclusions: In male patients older than 75 years with PSA $>30 \mathrm{ng} / \mathrm{ml}$ or with PSA $>20 \mathrm{ng} / \mathrm{ml}$ and suspicious digital rectal examination, histological confirmation of carcinoma by prostatic biopsy may be not necessary, because of the high probability of a positive result. When histological confirmation of prostatic carcinoma is required in elderly patients, transrectal prostatic FNA is a valid alternative to transrectal biopsy, due to its excellent tolerance and low complication rate.

Keywords: Prostatic carcinoma. Elderly patients. FNA. Biopsy. PSA. 
$\mathrm{L}^{2}$ a introducción clínica del antígeno prostático específico (PSA) como marcador sérico ha permitido el desarrollo de programas de cribaje y diagnóstico precoz del cáncer de próstata. Aunque el cribaje permite adelantar el diagnóstico del cáncer de próstata al menos 10 años ${ }^{1-4}$, sus beneficios potenciales son aún controvertidos. Dos estudios en curso, uno europeo y otro americano, pretenden demostrar definitivamente si el cribaje con PSA reduce la mortalidad por cáncer de próstata, pero sus resultados no estarán disponibles hasta dentro de 1-2 años ${ }^{5,6}$. Por el contrario, el cribaje indiscriminado del cáncer de próstata conlleva un riesgo de sobrediagnóstico y sobretratamiento, ya que permite detectar cánceres de pequeño volumen y escasa significación clínica, en los que la vigilancia activa puede ser una buena alternativa a la cirugía radical $^{7,8}$.

Esta situación es particularmente relevante en pacientes de edad avanzada. Se ha comprobado que los pacientes ancianos en los que en los que se detecta un cáncer de próstata mediante cribaje rara vez mueren por esta enfermedad en los 15 años siguientes al diagnóstico a pesar de no seguir tratamiento $^{9,10}$. De hecho, los beneficios del cribaje del cáncer de próstata parecen disminuir drásticamente con la edad, sobre todo a partir de los 65 años ${ }^{11}$.

Además, la biopsia prostática transrectal no está exenta de complicaciones, que parecen ser más frecuentes y más graves en la población anciana. En algunas series, hasta un $65 \%$ de las biopsias presentaron complicaciones, requiriéndose hospitalización en más del $4 \%^{12-14}$. Incluso hay recogidas en la literatura dos muertes por sepsis tras biopsia prostática ${ }^{15}$.

Por todo ello, en algunos estudios se ha comenzado a cuestionar la indicación de la biopsia prostática transrectal en pacientes octogenarios con un alto gado de sospecha clínica de cáncer de próstata (PSA >30 ng/ml y/o tacto rectal sospechoso), dada la alta tasa de detección de cáncer de próstata en esta población (cercana al 100\%) y el escaso impacto de los datos pronósticos del estudio histológico (grado de diferenciación, porcentaje de tumor en el material biopsiado) sobre las decisiones terapéuticas en estos pacientes ${ }^{16,17}$.

Una alternativa a la biopsia prostática transrectal es el estudio citológico del material obtenido mediante punción-aspiración con aguja fina (PAAF), guiada mediante tacto rectal. Esta técnica desarrollada hace más de 40 años por Esposti ${ }^{18}$ llegó a España más tarde ${ }^{19}$, cumpliendo un papel en el diagnóstico del cáncer de próstata antes de la generalización de las biopsias transrectales mediante tru-cut. Actualmente, este procedimiento sigue siendo ampliamente utilizado en los países escandinavos incluso en pacientes jóvenes ${ }^{20} \mathrm{y}$, dada su buena tolerancia y baja incidencia de complicaciones, parece particularmente adecuada para pacientes ancianos o con mal estado general. No obstante, el diagnóstico de adenocarcinoma mediante PAAF requiere que éste sea realizado por un citólogo con experiencia en la técnica ${ }^{21}$.

En el presente estudio se pretende analizar si es posible evitar biopsias de próstata en la población anciana, planteándose los siguientes objetivos:

1. Analizar la frecuencia del diagnóstico de cáncer de próstata mediante PAAF transrectal de próstata en varones ancianos con sospecha clínica de cáncer de próstata.

2. Identificar si existe algún grupo de varones ancianos con sospecha clínica de cáncer de próstata, en el cual la confirmación anatomopatológica del diagnóstico puede no ser necesaria.

3. Valorar si la PAAF transrectal de próstata es una técnica adecuada para el estudio de la población anciana analizando su tolerancia clínica y tasa de complicaciones.

\section{MATERIAL Y MÉTODOS}

Se trata de un estudio prospectivo en el cual desde enero de 2006 hasta marzo de 2007 se evaluaron mediante PAAF prostática 72 pacientes con edades comprendidas entre 75 y 93 años. Las 72 punciones fueron realizadas por 2 urólogos asistidos por un anatomopatólogo.

A todos los pacientes se les realizó una historia clínica, una exploración física con tacto rectal, y al menos una determinación del PSA sérico. Se consideró como indicación para realizar biopsia o PAAF prostática un tacto rectal sospechoso de neoplasia y/o un PSA superior a $4 \mathrm{ng} / \mathrm{ml}$.

Una vez se le indicaba al paciente la necesidad de realizarle la prueba, se le informaba verbalmente de la técnica y se le entregaba para su firma un consentimiento informado con todas las características técnicas y posibles complicaciones. Se les indicaba también que no vinieran en ayunas pero que no 
desayunaran de manera copiosa. Igualmente se les dio por escrito las instrucciones para la preparación del tracto intestinal y que consisten en la aplicación de dos enemas de limpieza, uno al acostarse la noche antes y otro a primera hora de la mañana del día de la prueba. En aquellos pacientes que tomaban anticoagulantes orales o antiagregantes plaquetarios no fue necesario suprimir previamente la medicación o sustituirla por heparinas de bajo peso molecular para la realización de la prueba.

La PAAF prostática se realizó según la técnica descrita por Esposti ${ }^{18}$.

El material necesario para realizar una PAAF es:

- Silla especial con perneras automatizada.

- Aguja para PAAF 22 G x 20.

- Portajeringas CAMECO de 10-20 ml.

- Jeringa de $20 \mathrm{ml}$.

- Dedil con guía para agujas.

- Portaobjetos.

Una vez en posición, se hizo una nueva exploración mediante tacto rectal. En nuestro estudio cada paciente fue sometido al menos a dos exámenes digitales por dos urólogos distintos coincidiendo casi en la práctica totalidad de los casos. En caso de discrepancia, se consideró que el tacto rectal era sospechoso de cáncer si al menos uno de los tactos rectales así lo consideraba. El resultado del tacto rectal fue considerado positivo si era sospechoso de cáncer o negativo si era normal o compatible con patología benigna.

Para realizar la PAAF se coloca el dedil en el dedo índice de la mano que va a localizar digitalmente los lóbulos prostáticos. Se coloca la aguja a través de la guía del dedil, se punciona la superficie prostática transrectalmente y se toman muestras para citología mediante aspiración del contenido. Las muestras extraídas fueron extendidas en portaobjetos y se realizó secado al aire, posteriormente se aplicó la tinción Diff-Quik“. Se valoró la existencia de células tumorales, en cuyo caso se evaluó el grado de diferenciación tumoral clasificándolo en: bien diferenciado, moderadamente diferenciado o mal diferenciado.

No fue necesaria la administración de ningún tipo de profilaxis antibiótica, ni la administración de analgesia para la realización de la prueba dada su buena tolerancia, y sólo se les recomendó que tomaran analgésicos si sufrían alguna molestia en el domicilio. Se advirtió a los pacientes de la posibili- dad de rectorragia y/o hematuria en las 24-48 horas siguientes a la prueba. De la misma manera también se les recomendó que si tuvieran fiebre o si el sangrado no remitía acudieran a Urgencias de nuestro hospital donde serían valorados por el urólogo de guardia.

Inicialmente se calculó la frecuencia total del diagnóstico citológico de cáncer de próstata, así como la de los diferentes grados de diferenciación. Para el estudio estadístico se calcularon las frecuencias de diagnósticos citológicos de cáncer próstata en función de la edad, tacto rectal (positivo o negativo) y niveles de PSA sérico. Para calcular las frecuencias según los niveles de PSA, estos fueron categorizados en 4 grupos: Menor de $10 \mathrm{ng} / \mathrm{ml}, 10-$ $20 \mathrm{ng} / \mathrm{ml}, 20-30 \mathrm{ng} / \mathrm{ml}$ y mayor de $30 \mathrm{ng} / \mathrm{ml}$. También se compararon los niveles de PSA sérico de las poblaciones con diagnóstico citológico de cáncer o con diagnóstico citológico de patología benigna mediante el test de Mann-Whitney.

Para analizar las frecuencias de diagnóstico de cáncer de próstata en función de ambas variables (tacto rectal y PSA) se confeccionaron 8 grupos, considerando las opciones tacto rectal positivo o negativo en cada uno de los grupos categóricos de niveles de PSA.

Finalmente se analizó mediante curva ROC el punto óptimo de corte de los niveles de PSA para el diagnóstico de cáncer de próstata en función de la sensibilidad y especificidad, analizando posteriormente las frecuencias relativas de diagnósticos citológicos de cáncer en los grupos de pacientes con niveles de PSA mayores o menores que el punto óptimo de corte. Posteriormente, se introdujo la variable tacto rectal en este análisis, calculando las frecuencias relativas del diagnóstico citológico de cáncer en las poblaciones con niveles de PSA mayor o menor que el punto de corte óptimo y con tacto rectal positivo o negativo.

\section{RESULTADOS}

La edad media de los 72 pacientes analizados fue de 82 años (75-93 años), con un PSA medio de $21,78 \mathrm{ng} / \mathrm{ml}$ (mediana 10,85, rango 5,2-226).

En 35 de estos pacientes $(48,6 \%)$ el resultado de la citología fue positivo para adenocarcinoma de próstata, mientras que en 37 fue negativo para tumor. A siete pacientes se les había realizado en años anteriores biopsia o PAAF transrectales que 
fueron negativas para tumor, pero se indicó realizar una nueva PAAF ante el aumento progresivo del PSA durante el seguimiento. El rango de PSA en estos casos fue de 9,5-57 ng/ml. En cuatro de los siete casos se encontraron células tumorales y sólo uno de ellos presentaba un tacto rectal sospechoso de malignidad.

El grado de diferenciación tumoral pudo establecerse en 29 de los 35 pacientes con cáncer, siendo la mayoría (69\%) adenocarcinomas bien diferenciados.

No se apreciaron diferencias en los niveles de PSA en función del grado de diferenciación tumoral. Así, el rango de PSA de los cánceres bien diferenciados fue de 5,8-47,6 ng/ml, el de los moderadamente diferenciados 6,2-207 ng/ml, y los valores de las 2 muestras clasificadas como mal diferenciados fueron de 25 y $226 \mathrm{ng} / \mathrm{ml}$. El intervalo de los PSA en los 6 pacientes en los que no se pudo determinar el grado citológico fue entre 7 y $98 \mathrm{ng} / \mathrm{ml}$.

$\mathrm{Al}$ analizar la frecuencia relativa de las citologías positivas en función la edad, no se observó diferencias en la misma en las distintas edades comprendidas en el rango analizado.

El 69,7\% de los pacientes con tacto rectal sospechosos y el 30,8\% de aquellos con tacto rectal negativo presentaron una citología positiva para cáncer (Tabla 1). Esto representa una sensibilidad para el tacto rectal de un 65,7\% y un especificidad del $73 \%$.

El valor medio del PSA en la población analizada fue $21,8 \mathrm{ng} / \mathrm{ml}$ (mediana 10,85, rango 5,2-226). De los 72 pacientes evaluados, cerca de la mitad (44\%) presentaban un PSA inferior a $10 \mathrm{ng} / \mathrm{ml}$. Dos pacientes eran portadores de sonda vesical permanente (PSA de 20 y 29,3 ng/ml) y un paciente había sido intervenido por hiperplasia prostática benigna 10 años antes (PSA 6,3 ng/ml); en los 3 casos el resultado de la PAAF fue negativo para tumor.

El PSA medio de los pacientes con PAAF positivas fue de $33,3 \mathrm{ng} / \mathrm{ml}$ (mediana 18, rango 5,8-226), $\mathrm{y}$ el de los pacientes con PAAF negativas de 10,9 $\mathrm{ng} / \mathrm{ml}$ (mediana 9,2, rango 5,2-29,3), siendo esta diferencia estadísticamente significativa $(p=0,0006$, test de Mann-Whitney).

Tabla 1. Resultados de la PAAF según tacto rectal

\begin{tabular}{lccc}
\hline & Tacto rectal + & Tacto rectal - & Total \\
\hline $\mathrm{PAAF}+$ & 23 & 12 & 35 \\
$\mathrm{PAAF}-$ & 10 & 27 & 37 \\
& 33 & 39 & 72 \\
\hline
\end{tabular}

La distribución de las citologías positivas según los niveles del PSA, categorizados en 4 grupos se recoge en la Tabla 2. Cabe destacar el aumento del porcentaje de citologías positivas para adenocarcinoma conforme aumenta el valor del PSA llegando al $80 \%$ para PSA entre 20 y $30 \mathrm{ng} / \mathrm{ml}$ y al $100 \%$ para pacientes con un PSA superior a $30 \mathrm{ng} / \mathrm{ml}$.

Al considerar conjuntamente los resultados del tacto rectal y del PSA se comprueba un aumento progresivo de la proporción de pacientes con PAAF positivas según aumenta el PSA, sobre todo en los casos en los que, además, el tacto es sospechoso de cáncer (Tabla 3). De hecho, el 100\% de los pacientes con PSA superior a $20 \mathrm{ng} / \mathrm{ml}$ con tacto rectal sospechoso, la PAAF fue positiva.

$\mathrm{Al}$ ser los valores de PSA una variable continua, se calculó la curva ROC para determinar el punto óptimo de corte con mejor sensibilidad y especificidad diagnósticas.

Los cálculos dieron como resultado que el valor de PSA donde se produce un equilibrio entre la máxima Sensibilidad-Especificidad era de 12,8 $\mathrm{ng} / \mathrm{ml}$ obteniendo una sensibilidad del $65,7 \%$ y una especificidad del 75,7 \% (Fig. 1). Al clasificar a los pacientes según el punto de corte óptimo de PSA $(12,8 \mathrm{ng} / \mathrm{ml})$ y los hallazgos del tacto rectal, se com-

Tabla 2. Resultados de la PAAF según el PSA

\begin{tabular}{lccc}
\hline PSA (ng/ml) & PAAF+ & PAAF- & Total \\
\hline$<10$ & $10(31 \%)$ & $22(69 \%)$ & 32 \\
$10-20$ & $10(43 \%)$ & $13(57 \%)$ & 23 \\
$20-30$ & $8(80 \%)$ & $2(20 \%)$ & 10 \\
$>30$ & $7(100 \%)$ & 0 & 7 \\
Total & 35 & 37 & 72 \\
\hline
\end{tabular}

Tabla 3. Resultados de la PAAF según el PSA y el tacto rectal

\begin{tabular}{lccc}
\hline & PAAF + & PAAF - & Total \\
\hline PSA<10, TR- & $7(30 \%)$ & $16(70 \%)$ & 23 \\
PSA<10, TR+ & $3(33 \%)$ & $6(67 \%)$ & 9 \\
PSA 10-20, TR- & $5(36 \%)$ & $9(64 \%)$ & 14 \\
PSA 10-20, TR+ & $5(56 \%)$ & $4(44 \%)$ & 9 \\
PSA 20-30, TR- & 0 & $2(100 \%)$ & 2 \\
PSA 20-30, TR+ & $8(100 \%)$ & 0 & 8 \\
PSA $>30$, TR- & 0 & 0 & 0 \\
PSA $>30$, TR+ & $7(100 \%)$ & 0 & 7 \\
Total & 35 & 37 & 72 \\
\hline
\end{tabular}




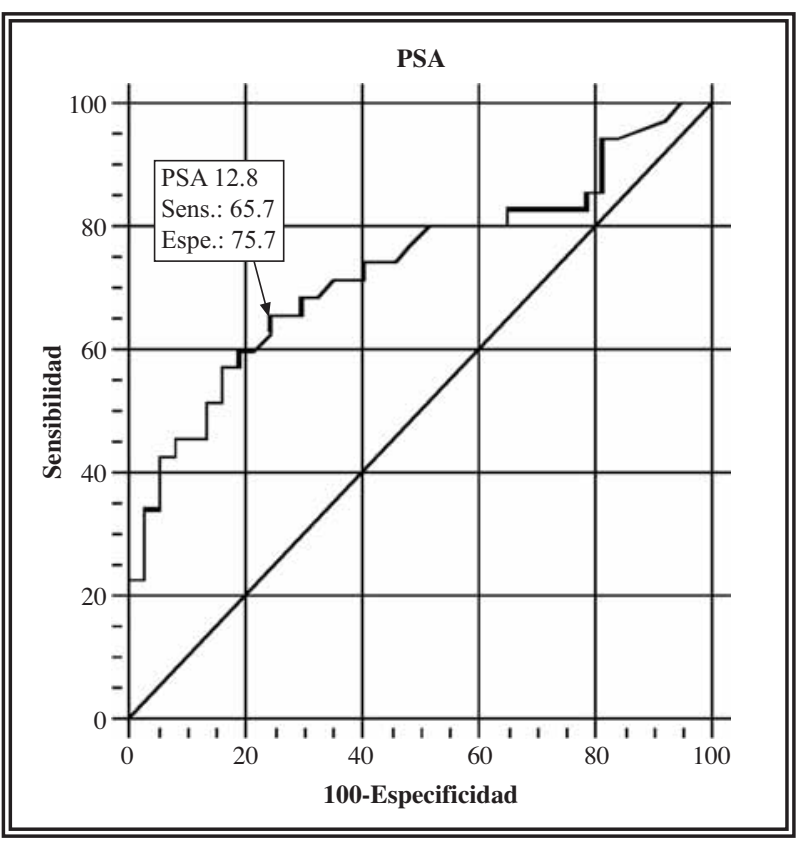

FIGURA 1. Curva ROC para los valores de PSA y PAAF positiva o negativa.

probó que el $90 \%$ de los pacientes con PSA superior a $12,8 \mathrm{ng} / \mathrm{ml}$ y tacto rectal positivo presentaban citologías positivas para cáncer (Tabla 4).

De los 72 pacientes a los que se les realizó la PAAF sólo uno tuvo hematuria macroscópica y precisó valoración por el urólogo de guardia, y 3 presentaron síndrome miccional con polaquiuria, disuria, tenesmo y hematuria. De estos 3 pacientes, sólo en un caso requirió ingreso en la unidad de corta estancia para el tratamiento de una prostatitis aguda, mientras que el resto pudieron ser remitidos a su domicilio en el mismo día con tratamiento antibiótico empírico. Cabe destacar que ningún paciente sufrió episodios vasovagales durante la realización de la prueba. Estos resultados suponen un $4,1 \%$ de complicaciones menores y un $1,3 \%$ de complicaciones mayores (entendiendo como tales aquellas que necesiten hospitalización) en nuestra serie.

Tabla 4. Resultados de la PAAF según resultados de la curva ROC

\begin{tabular}{lccc}
\hline & $\begin{array}{c}\text { PAAF+ } \\
\mathbf{n}(\%)\end{array}$ & $\begin{array}{c}\text { PAAF- } \\
\mathbf{n}(\%)\end{array}$ & Total \\
\hline PSA $>12,8$, TR+ & $19(90)$ & $2(10)$ & 21 \\
PSA $>12,8$, TR- & $4(37)$ & $7(63)$ & 11 \\
PSA $<12,8$, TR+ & $4(33)$ & $8(67)$ & 12 \\
PSA $<12,8$, TR- & $8(29)$ & $20(71)$ & 28 \\
Total & 35 & 37 & 72 \\
\hline
\end{tabular}

\section{DISCUSIÓN}

El 49\% (35/72) de los pacientes analizados en nuestro estudio fueron diagnosticados de carcinoma de próstata, manteniéndose esta proporción si consideramos únicamente a los octogenarios (50\%, 33/66). En un estudio similar realizado por Bott et al. ${ }^{16}$ la tasa de detección de cáncer de próstata en varones mayores de 80 años fue superior a la nuestra (79\%). Independientemente de que esta serie inglesa es mucho más amplia (205 pacientes frente a 72) al tratarse de un estudio multicéntrico, dos factores pueden explicar los diferentes resultados.

Por una parte, en la serie inglesa se incluyen pacientes con niveles de PSA más elevados que los nuestros: PSA mayor o igual a $20 \mathrm{ng} / \mathrm{ml}$ en $101 \mathrm{de}$ los 205 pacientes (49\%) frente a 17 de 72 (24\%) en nuestra serie. Es decir, que probablemente se trataba de tumores en estadios más avanzados que los nuestros y, por lo tanto con mayor volumen tumoral, lo cual les hace ser más fácilmente detectables mediante las biopsias transrectales. Si consideramos únicamente los pacientes con PSA mayor de 20 $\mathrm{ng} / \mathrm{ml}$, la proporción de pacientes con cáncer de próstata es similar: 92\% (93/101) frente a $88 \%$ (15/17) en nuestra serie,

Otro factor a tener en cuenta es la diferente técnica empleada para el diagnóstico del cáncer de próstata: PAAF transrectal en nuestra serie y biopsia transrectal mediante tru-cut en la serie de Bott et al. ${ }^{16}$. Podría interpretarse que la PAAF es menos sensible para la detección del cáncer de próstata que la biopsia, Sin embargo, no parece ser éste el caso, ya que aunque no hemos realizado un estudio comparativo entre ambas técnicas, nuestros resultados y los de la serie inglesa son similares en cuanto a tasas de detección cuando se estratifica a los pacientes según los niveles de PSA sérico.

En cualquier caso, nuestros resultados sugieren que, en general, ante una sospecha clínica de cáncer de próstata a partir de los datos del PSA sérico y el tacto rectal en la población de dad avanzada, es necesaria la confirmación histológica o citológica del diagnóstico, ya que sólo en aproximadamente la mitad de estos pacientes se confirma el diagnóstico clínico.

Otra cuestión distinta es si en estos pacientes de edad avanzada asintomático o con sólo un síndrome de prostatismo leve o moderado esta justificado el realizar un diagnóstico precoz de cáncer de próstata. 
En España, esta situación no está claramente tipificada, y mientras algunos hospitales no realizan maniobras invasivas en pacientes mayores de 75 años, en otros todavía se reciben solicitudes de biopsias a pacientes de más de 80 años para determinar la aplicación de un posible tratamiento. Cabe destacar que dentro de estas solicitudes, se encuentran aquellas que por presiones del paciente o de la familia, debido a un desconocimiento de la enfermedad y la comprensible cancerofobia, se demanda una prueba de seguridad diagnóstica. Al analizar nuestros resultados en los pacientes con PSA mayor o igual a $20 \mathrm{ng} / \mathrm{ml}$, la PAAF confirmó el diagnóstico de cáncer de próstata en el $88 \%$ de los casos (15/17), porcentaje que aumenta hasta el $100 \%$ en los casos con PSA mayor o igual a 30 $\mathrm{ng} / \mathrm{ml}(7 / 7)$. Si se asocian los resultados del tacto rectal, en el 90\% de los pacientes con PSA > 12,8 $\mathrm{ng} / \mathrm{ml}$ y tacto rectal sospechoso (19/21), y en la totalidad de pacientes con PSA mayor o igual a 20 $\mathrm{ng} / \mathrm{ml}$ y tacto rectal sospechoso de cáncer (15/15), se confirmó el diagnóstico mediante PAAF. Estos resultados están en la línea de los obtenidos en el estudio de Bott et $\mathrm{al}^{16}$.

Aunque, evidentemente, es necesario estudiar una serie más amplia para confirmar estos hallazgos, estos resultados sugieren que en estos dos subgrupos de pacientes:

a) Varones mayores de 75 años con PSA > 30 $\mathrm{ng} / \mathrm{ml}$.

b) Varones mayores de 75 años con PSA > 20 $\mathrm{ng} / \mathrm{ml}$ y tacto rectal sospechoso de cáncer cabrían dos alternativas:

La primera de ellas sería basarse en los datos clínicos para establecer el diagnóstico y evitar así la biopsia de próstata y sus complicaciones. Las opciones terapéuticas serían:

- la observación y vigilancia de la evolución del PSA y la aparición de síntomas en los pacientes asintomático, o

- la instauración precoz de un tratamiento hormonal en los que presenten sintomatología $^{20,22,23}$. Esta es la alternativa propuesta por el grupo inglés ${ }^{16}$.

Otra posibilidad sería confirmar el diagnóstico mediante PAAF transrectal como hemos realizado en nuestros pacientes. Aunque la probabilidad del diagnóstico de cáncer de próstata es muy alta, la PAAF es una técnica bien tolerada incluso en esta población de edad avanzada y con una baja tasa de complicaciones. Aunque no permite clasificar a los pacientes según el grado de Gleason y el porcentaje de tumor en los cilindros obtenidos mediante biopsia con tru-cut, estos parámetros no van a influir decisivamente en las indicaciones terapéuticas en esta población, ya que éstas se van a basar fundamentalmente en la existencia o no de sintomatología.

En nuestra serie la PAAF transrectal presentó una tasa de complicaciones baja. Solamente 4 pacientes refirieron alguna complicación, y, de ellos, únicamente un caso sufrió una prostatitis aguda que requirió ingreso durante 24 horas, evolucionando favorablemente. Esta tasa de complicaciones es sensiblemente inferior a la comunicada tras la biopsia transrectal con tru-cut ${ }^{12,13,16}$. Además, la buena tolerancia de la prueba permite realizarla sin la administración de analgesia con mínimas molestias. En ningún caso se presentaron episodios vasovagales, que son relativamente frecuentes durante la realización de las biopsias mediante tru-cut. Y dada la poca agresividad de la PAAF, optamos por los enemas de limpieza antes de la prueba como única medida profiláctica sin la administración de antibióticos. También tiene como ventaja, dada su baja agresividad, que no se necesita retirar o modificar los posibles tratamientos anticoagulantes o antiagregantes antes de realizar la prueba.

Por lo tanto los resultados de nuestro estudio sugieren que:

1. Ante una sospecha clínica de cáncer de próstata a partir de los datos del PSA sérico y tacto rectal en la población mayor de 75 años, es necesaria la confirmación histológica o citológica del diagnóstico, ya que sólo en aproximadamente la mitad de estos pacientes se confirma el diagnóstico clínico.

2. En varones mayores de 75 años con PSA $>30$ $\mathrm{ng} / \mathrm{ml}$ o con PSA > $20 \mathrm{ng} / \mathrm{ml}$ y tacto rectal sospechoso de cáncer puede no ser necesaria la confirmación histológica de cáncer de próstata mediante biopsia transrectal, dada la elevada probabilidad de que ésta sea positiva.

3. Cuando se considere necesario o conveniente la confirmación anatomopatológica del diagnóstico de cáncer de próstata en pacientes de edad avanzada, la PAAF transrectal constituye una buena alternativa a la biopsia transrectal con trucut, dada su excelente tolerancia y baja tasa de complicaciones. 


\section{REFERENCIAS}

1. Draisma G, Boer R, Otto SJ, van der Cruijsen IW, Damhuis RA, Schröder FH, et al. Lead times and overdetection due to prostate-specific antigen screening: estimates from the European Randomized Study of Screening for Prostate Cancer. J Natl Cancer Inst. 2003;95(12):868-878.

2. Stamey TA, Yang N, Hay AR, McNeal JE, Freiha FS, Redwine E. Prostate specific antigen as a serum marker for adenocarcinoma of the prostate. N Engl J Med. 1987;317(15):909-916.

3. Luján Galán M, Páez Borda A, Chiva Robles V, Santonja Garriga C, Romero Cajigal I, Berenguer Sánchez A. Tendencias epidemiológicas en cáncer de próstata durante los últimos años. Arch Esp Urol. 2004;57(8):817-825.

4. Catalona WJ, Richie JP, Ahmann FR, Hudson MA, Scardino PT, Flanigan RC, et al. Comparasion of digital rectal exam and serum prostate specific antigen in the early detection of prostate cancer: results of a multicenter clinical trial of 6630 men. J Urol. 2004; 151(5): 1283-1290.

5. Prorock PC, Andriole GL, Bresalier RS et al. Prostate, Lung, Colorectal and Ovarian Cancer Screening Trial Project Team. Design of the Prostate, Lung, Colorectal and Ovarian (PLCO) Cancer Screening Trial. Control Clin Trials. 2000; 21: 273S309S.

6. De Konig HJ, Liem MK, Baan CA et al. Prostate cancer mortality reduction by screening: power and time frame with complete enrolment in the European Randomised Screening for Prostate Cancer (ERSPC) trial. Int J Cancer. 2002;98: 268-273.

7. Van der Cruijsen-Koeter IW, Vis AN, Roobol MJ, Wildhagen MF, de Koning HJ, van der Kwast TH, et al. Comparison of screen detected and clinically diagnosed prostate cancer in the European randomized study of screening for prostate cancer, section Rotterdam. J Urol. 2005;174(1):121-125.

8. Klotz LH, Nam RK. Active surveillance with selective delayed intervention for favorable risk prostate cancer: clinical experience and a 'number needed to treat' analysis. Can J Urol. 2006;13 Suppl 1:48-55.

9. Fleming C, Wasson JH, Albertsen PC, Barry MJ, Wennberg JE. A decision analysis of alternative treatment strategies for clinically localized prostate cancer. Prostate Patient Outcomes Research Team. JAMA. 1993;269(20):2650-2658.

10. Johansson JE, Andrén O, Andersson SO, Dickman PW, Holmberg L, Magnuson A, et al. Natural history of early, localized prostate cancer. JAMA. 2004;291(22):2713-2719.

11. Ross KS, Guess HA, Carter HB. Estimation of treatment benefits when PSA screening for prostate cancer is discontinued at different ages. Urology. 2005;66(5):1038-1442.

12. Rodriguez LV, Terris MK. Risks and complications of transrectal ultrasound guided prostate needle biopsy: a prospective study and review of the literature. J Urol. 1998; 160(6 Pt 1): 2115-2120.

13. Lindert KA, Kabalin JN, Terris MK. Bacteraemia and bacteriuria after transrectal ultrasound guided prostate biopsy. J Urol. 2000;164(1):76-80.
14. Crawford ED, Haynes AL Jr, Story MW, Borden TA. Prevention of urinary tract infection and sepsis following transrectal prostate biopsy. J Urol. 1982;127(3):449-451.

15. Brewster SF, Rooney N, Kabala J, Feneley RC. Fatal anaerobic infection following transrectal biopsy of a rare prostatic tumor. Brit J Urol. 1993;72(6):977-978.

16. Bott SR, Foley CL, Bull MD, Reddy CC, Freeman A, Montgomery BS,et al. Are prostatic biopsies necessary in men aged $\geq 80$ years?. BJU Int. 2007;99(2):335-338.

17. Draisma G, Boer R, Otto SJ, van der Cruijsen IW, Damhuis RA, Schröder FH, et al. Lead times and overdetection due to prostate-specific antigen screening: estimates from the European Randomized Study of Screening for Prostate Cancer. J Natl Cancer Inst. 2003;95(12):868-878.

18. Esposti PL. Cytologic diagnosis of prostatic tumors with the aid of transrectal aspiration biopsy. A critical review of 1110 cases and a report of morphologic and cytochemical studies. Acta Cytol. 1966;10(3):182-186.

19. Cervell Ferrón JM, Cuevas de Aldasoro ML, García Pérez R, Chacón Gómez E, Lescure del Río S, Pérez-Guillermo García M. Citología aspirativa de próstata: su utilidad diagnóstica y repercusión sobre el coste de la asistencia. Actas Urol Esp. 1989;13(1):28-31.

19. Cervell Ferrón JM, Cuevas de Aldasoro ML, García Pérez R, Chacón Gómez E, Lescure del Río S, Pérez-Guillermo García M. Aspirative cytology of the prostate: its diagnostic usefulness and effect on care costs. Actas Urol Esp. 1989;13(1):28-31.

20. Heindenreich A, Aus G, Abbou CC et al. Guidelines on Prostate Cancer. EAU Guidelines. 2007.

21. Pérez-Guillermo M, Acosta-Ortega J, García-Solano J. Pitfalls and infrequent findings in fine-needle aspiration of the prostate gland. Diagn Cytopathol. 2005;33(2):126-37.

22. Medical Research Council Prostate Cancer working Party Investigations Group. Immediate versus deferred treatment for advanced prostatic cancer. Initial results of the Medical Research Council Trial. Br J Urol. 1997;79(2):235-246.

23. Studer UE, Whelan P, Albrecht W, Casselman J, de Reijke T, Hauri D, et al. Immediate or deferred androgen deprivation for patients with prostate cancer not suitable for local treatment with curative intent. European Organisation for Research and Treatment of Cancer Trial 30891. J Clin Oncol. 2006;24(12): 1868-1876.

Correspondencia autor: Dr. J. Planelles Gómez

Servicio de Urología

Hospital Universitario Dr. Peset

Gaspar Aguilar, 90 - 46017 Valencia

Tel.: 963862500

E-mail autor: jorge_planelles@yahoo.es

Información artículo: Original - Cáncer de próstata

Trabajo recibido: enero 2008

Trabajo aceptado: febrero 2008 